\title{
Modeling and Characterization of SiGe HBT Low-Frequency Noise Figures-of-Merit for RFIC Applications
}

\author{
Jin Tang, Student Member, IEEE, Guofu Niu, Senior Member, IEEE, Zhenrong Jin, Student Member, IEEE, \\ John D. Cressler, Fellow, IEEE, Shiming Zhang, Student Member, IEEE, Alvin J. Joseph, Member, IEEE, and \\ David L. Harame, Senior Member, IEEE
}

\begin{abstract}
We present the first systematic experimental and modeling results of noise corner frequency $\left(f_{C}\right)$ and noise corner frequency to cutoff frequency ratio $\left(f_{C} / f_{T}\right)$ for SiGe heterojunction bipolar transistors (HBTs) in a commercial SiGe RF technology. The $f_{C}$ and $f_{C} / f_{T}$ ratio are investigated as a function of operating collector current density, SiGe profile, breakdown voltage, and transistor geometry. We demonstrate that both the $f_{C}$ and $f_{C} / f_{T}$ ratio can be significantly reduced by careful SiGe profile optimization. A comparison of the $f_{C}$ and $f_{C} / f_{T}$ ratio for high breakdown and standard breakdown voltage devices is made. Geometrical scaling data show that the SiGe HBT with $A_{E}=0.5 \times 2.5 \mathrm{\mu m}^{2}$ has the lowest $f_{C}$ and $f_{C} / f_{T}$ ratio compared to other device geometries. An $f_{C}$ reduction of nearly $50 \%$ can be achieved by choosing this device as the unit cell in RF integrated-circuit design.
\end{abstract}

Index Terms-Breakdown voltage, corner frequency, cutoff frequency, device modeling, flicker noise, heterojunction bipolar transistor (HBT), low-frequency noise, phase noise, RF integrated circuit (RFIC), SiGe.

\section{INTRODUCTION}

$\mathbf{S}$ iGe heterojunction bipolar transistor (HBT) technology has come of age as an important semiconductor technology for both wired and wireless telecommunications applications because of its superior analog and RF performance, together with its CMOS integration capability [1]. By employing bandgap engineering, SiGe HBTs outperform Si bipolar junction transistors (BJTs) in nearly every important performance metric and, in several areas, provide improved performance over the III-V HBTs. One of the areas in which SiGe HBTs exceed GaAs HBTs is in low $1 / f$ noise corner frequency [2], thereby making

Manuscript received April 4, 2002. This work was supported by the National Science Foundation under Grant ECS-0 119623 and Grant ECS-0112923, by the Semiconductor Research Corporation under Grant SRC 2001-NJ-937 and Grant 2000-HJ-769, by IBM under a Faculty Partnership Research Award, and by the Alabama Microelectronics Science and Technology Center.

J. Tang, G. Niu, and S. Zhang are with the Alabama Microelectronics Science and Technology Center, Electrical and Computer Engineering Department, Auburn University, Auburn, AL 36849 USA (e-mail: tangjin@eng.auburn.edu).

$<Z$. Jin affiliation must match current biography affiliation. Pls. correct.> Z. Jin and J. D. Cressler were with the Alabama Microelectronics Science and Technology Center, Electrical and Computer Engineering Department, Auburn University, Auburn, AL 36849 USA. They are now with the School of Electrical and Computer Engineering, Georgia Institute of Technology, Atlanta, GA 30332-0250 USA.

A. J. Joseph and D. L. Harame are with IBM Microelectronics, Essex Junction, VT 05452 USA.

Publisher Item Identifier 10.1109/TMTT.2002.804519. them an excellent choice for low-noise amplifiers, oscillators [3], and power amplifiers.

The traditional figure-of-merit for low-frequency noise, the noise corner frequency $\left(f_{C}\right)$, accounts for only $1 / f$ noise. In circuit design, however, the speed of the transistors is also a critical design issue. Si BJTs typically have low $f_{C}$, but do not have sufficient gain to sustain oscillation at RF and microwave frequencies because of their limited $f_{T}$. GaAs HBTs have high $f_{T}$, but typically have high $f_{C}$ and, hence, generate larger phase noise when used in oscillators. SiGe HBTs, however, provide $f_{T}$ comparable to GaAs HBTs and lower $f_{C}$ than Si BJTs, making them an attractive choice for ultra-low phase-noise oscillators [3]. A better figure-of-merit to characterize low-frequency noise for these applications is the $f_{C} / f_{T}$ ratio [4], originally defined in [5], because it takes into account high-frequency response through $f_{T}$.

This paper presents modeling and experimental results of the low-frequency noise figures-of-merit $f_{C}$ and $f_{C} / f_{T}$ in a commercial SiGe RF technology. Low-frequency noise spectra and high-frequency $s$-parameters were measured, from which $f_{C}$ and $f_{T}$ are extracted. Four SiGe HBT wafers featuring different SiGe profile designs were used to examine the impact of SiGe profile shape on $f_{C}$ and $f_{C} / f_{T}$. The profile comparison results are then used to derive a new figure-of-merit for SiGe profile design. Many wireless systems operate at frequencies below $50 \mathrm{GHz}$, the peak $f_{T}$ achieved in this SiGe technology. The excess $f_{T}$ can be traded for higher breakdown voltage to allow larger signal power, which reduces additive phase noise in voltage-controlled oscillators [6]. We will examine how the fabrication process modifications required for increasing breakdown voltage affects low-frequency noise, which then determines modulative phase noise in oscillators.

To facilitate optimum choice of biasing current in circuit design, $f_{C}$ and $f_{T}$ were measured across a wide range of operating current. One of the levers in RF integrated-circuit (RFIC) design is that the device size and layout can be used as design variables. Emitter length, for example, can be optimized for active noise matching in low-noise amplifier (LNA) design [7]. The same total device area required is typically obtained by parallel connection of small area unit cells. An array of SiGe HBT unit cells with different emitter areas are characterized over a wide range of biasing current to facilitate the optimum choice of device geometry, biasing current, and unit cell size that minimizes the low-frequency noise. 


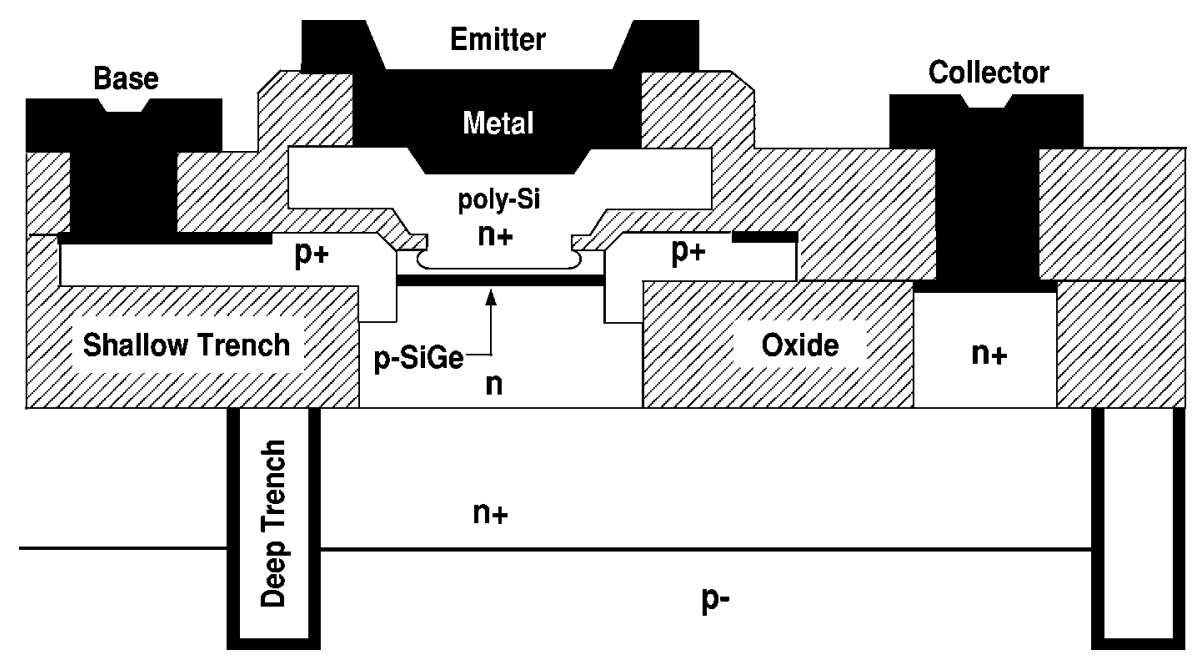

Fig. 1. Schematic cross section of the SiGe HBTs used in this study.

\section{Device Technology}

Fig. 1 shows a schematic cross section of the SiGe HBT used in this study. The SiGe HBT has a planar self-aligned structure with a conventional polyemitter contact, silicided extrinsic base, and deep- and shallow-trench isolation. The SiGe base was grown using the ultrahigh vacuum/chemical vapor deposition (UHV/CVD) technique. Devices of two different breakdown voltages were obtained on the same chip in the same fabrication flow by selective implantation during collector formation. The standard breakdown voltage (SBV) devices received both a deep and a shallow collector implant, and have a peak $f_{T}$ of $50 \mathrm{GHz}(\mathrm{BV} \mathrm{CEO}=3.3 \mathrm{~V})$. The high breakdown voltage (HBV) devices received only the deep collector implant, and have a peak $f_{T}$ of $30 \mathrm{GHz}\left(\mathrm{BV}_{\mathrm{CEO}}=5.3 \mathrm{~V}\right)$. Details of the fabrication process can be found in [8].

Four wafers with different $\mathrm{SiGe}$ profile designs were measured, including a $10 \%$ peak SiGe control, a $14 \%$ peak low-noise design ( $L N 1)$, a $18 \%$ peak low-noise design ( $L N 2)$, and an $\mathrm{Si}$ BJT comparison. Details of the SiGe profile design can be found in [9] and [10]. All of the wafers were fabricated in the same wafer lot under identical processing conditions. The SiGe films in all of the SiGe designs are unconditionally stable to defect generation. Compared to the SiGe control, the $L N 1$ and $L N 2$ designs have a higher Ge content and a larger Ge gradient in the neutral base to achieve higher $\beta$ and higher $f_{T}$, but less Ge retrograding into the collector to keep the total Ge content within the thermal stability limit.

\section{LOW-FREQUENCY NOISE FIGURES-OF-MERIT}

It has been experimentally established that the major $1 / f$ noise source in these SiGe HBTs is the base current $1 / f$ noise [2], [3]. The $1 / f$ noise is proportional to $I_{B}^{\alpha}$ and inversely proportional to the emitter area $A_{E}$ as follows:

$$
S_{I_{B}}=\frac{K}{A_{E}} I_{B}^{\alpha} \frac{1}{f}
$$

where $K$ is a technology dependent constant, and $\alpha \approx 2$ for typical SiGe HBTs. $K / A_{E}$ corresponds to the flicker noise con- stant $K_{F}$ in SPICE. The noise corner frequency $f_{C}$ is obtained by equating the $1 / f$ noise $S_{I_{B}}$ to the shot noise $2 q I_{B}$ as follows:

$$
f_{C}=\frac{K I_{B}}{2 q A_{E}}=\frac{K J_{C}}{2 q \beta}
$$

where $\alpha=2$ is assumed for simplicity and insight, as detailed below, $J_{C}$ is the collector current density, and $\beta$ is the dc current gain.

Equation (2) suggests that $f_{C}$ is proportional to $J_{C}$ and $K$, and inversely proportional to $\beta$. This differs from that derived in [5]. The derivation of [5] showed that $f_{C}$ is independent of biasing current density because $\alpha=1$ was assumed according to mobility fluctuation. This, however, is not the case in our devices, which all show an $\alpha$ close to two.

The figure-of-merit for frequency response, cutoff frequency $f_{T}$, is related to $J_{C}$ by

$$
\frac{1}{2 \pi f_{T}} \approx \tau_{F}+\frac{1}{g_{m}} C_{t}=\tau_{F}+\frac{V_{t}}{J_{C}} C_{t}
$$

where $\tau_{F}$ is the forward transit time, $V_{t}=k T / q$ is the thermal voltage, $g_{m}=J_{C} / V_{t}$ is the transconductance per unit area, and $C_{t}$ is the total junction depletion capacitance per unit area. Prior to $f_{T}$, rolloff at high $J_{C}, \tau_{F}$, and $C_{t}$ are constant in the typical $J_{C}$ range of interest to $\mathrm{RF}$ circuits $\left(0.1-1.5 \mathrm{~mA} / \mu \mathrm{m}^{2}\right)$. The $f_{C} / f_{T}$ ratio is obtained by combining (2) and (3) as follows:

$$
\frac{f_{C}}{f_{T}}=K \frac{\pi}{q} \frac{J_{C}}{\beta}\left(\tau_{F}+V_{t} \frac{C_{t}}{J_{C}}\right)=\frac{K \pi}{\beta q}\left(\tau_{F} J_{C}+V_{t} C_{t}\right)
$$

Thus, the model suggests a linear increase of the $f_{C} / f_{T}$ ratio with operating collector current density $J_{C}$ provided that $\beta$ and $\tau_{F}$ are constant. This is in contrast to the prediction of a $J_{C}$ independent $f_{C} / f_{T}$ ratio in [5], which assumed $\alpha=1(\alpha \approx 2$ in our devices). At higher $J_{C}$, where $f_{T}$ is larger, $\tau_{F} J_{C} \gg$ $V_{t} C_{t}$ and $f_{C} / f_{T} \approx K \pi \tau_{F} J_{C} / \beta q$. Thus, the $f_{C} / f_{T}$ ratio is determined by the $K \tau_{F} / \beta$ term at higher $J_{C}$. A smaller $\tau_{F}$, higher $\beta$, and smaller $K$ factor are desired to reduce $f_{C} / f_{T}$. A smaller $f_{C} / f_{T}$ indicates better phase noise performance at higher frequencies. 


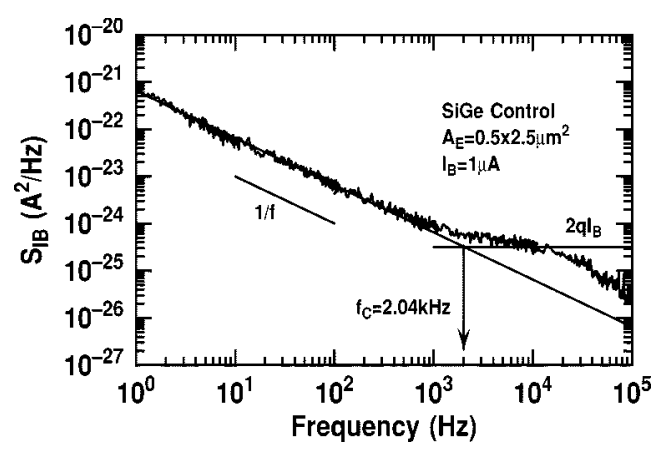

Fig. 2. Typical low-frequency noise spectrum of SiGe HBT used in this study $\left(A_{E}=0.5 \times 2.5 \mu \mathrm{m}^{2}, I_{B}=1 \mu \mathrm{A}\right)$.

\section{EXPERIMENTAL RESULTS}

Low-frequency noise spectra and $s$-parameters were measured on both standard and HBV devices for the SiGe control, the $L N 1$ and $L N 2$ low-noise SiGe designs, and the Si BJT comparison. Low-frequency noise was measured using an EG\&G 5113 preamplifier and an HP3561A dynamic signal analyzer controlled by a Labview program. $S$-parameters were measured from 0.5 to $40 \mathrm{GHz}$ using an HP8510C vector network analyzer, from which $f_{T}$ was extracted. The forward transit time $\tau_{F}$ and the depletion capacitance per unit area $C_{t}$ were determined from the intercept and slope of the linear extrapolation of the measured $1 / f_{T}-1 / J_{C}$ data, respectively. In the low-frequency noise measurements, devices were biased at collector current densities from 0.1 to $1.5 \mathrm{~mA} / \mu \mathrm{m}^{2}$, the range of interest to RF circuits for the SBV devices.

Fig. 2 shows a typical low-frequency base current noise spectrum $\left(S_{I_{B}}\right)$ for an SBV SiGe control HBT. The noise spectrum shows a clear $1 / f$ component and the $2 q I_{B}$ shot noise level. The corner frequency $f_{C}$ is determined from the intersection of the $1 / f$ component and the $2 q I_{B}$ shot noise level. The rolloff above $10 \mathrm{kHz}$ is due to the bandwidth limitation of the preamplifier used. The measured $S_{I_{B}} \times f$ product was plotted as a function of $I_{B}$, from which the SPICE $1 / f$ noise constant $K_{F}$ was extracted by assuming $\alpha=2$. The obtained $K_{F}$ is approximately proportional to $1 / A_{E}$, leading to an emitter area independent $K$ factor of $2.0 \times 10^{-9} \mu \mathrm{m}^{2}$. The measured $K$ factor is approximately the same for all of the SiGe designs.

\section{A. Collector Operating Current Dependence}

The measured and calculated $f_{C}$ versus $J_{C}$ are shown on the left $y$-axis of Fig. 3 for an SBV HBT on the SiGe control wafer. The measured $f_{T}$ versus $J_{C}$ dependence is shown on the right $y$-axis. The cutoff frequency $f_{T}$ increases with $J_{C}$ according to (3) prior to the $f_{T}$ rolloff at high injection. The corner frequency $f_{C}$ increases with $J_{C}$, as predicted by (2). The common practice of quoting corner frequency value without specifying biasing current and device geometry can be misleading because $f_{C}$ strongly depends on biasing current density, as shown by the data in Fig. 3. The calculated $f_{C}$ is in close agreement with measured data. The slight deviation from a linear increase is caused by the $J_{C}$ dependence of $\beta$ and the deviation of $\alpha$ from two. Fig. 4 shows the measured $f_{C} / f_{T}$ ratio, together with modeling results calculated using (4). The modeling results agree well with the measured data. The $f_{C} / f_{T}$ ratio increases with

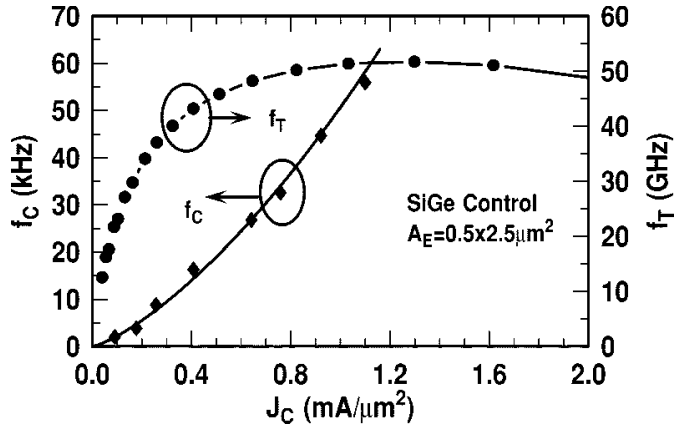

Fig. 3. Measured corner frequency $f_{C}$ and cutoff frequency $f_{T}$ as a function of $J_{C}$ for the SBV SiGe control HBT $\left(A_{E}=0.5 \times 2.5 \mu \mathrm{m}^{2}\right)$.

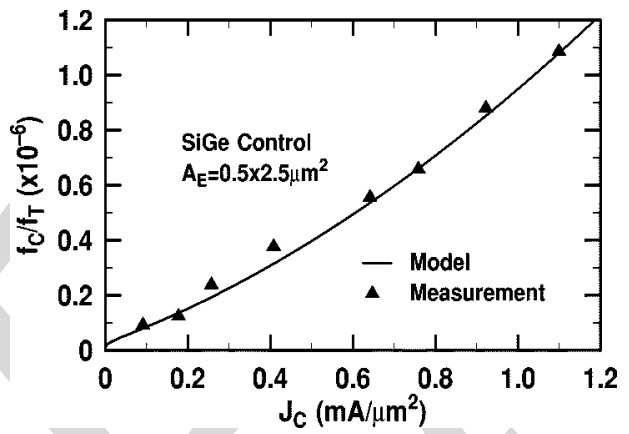

Fig. 4. Measured and modeled $f_{C} / f_{T}$ ratio as a function of $J_{C}$ for the SBV SiGe control HBT $\left(A_{E}=0.5 \times 2.5 \mu \mathrm{m}^{2}\right)$.

$J_{C}$, as predicted by (4). These results suggest that in order to reduce the $f_{C}$ and $f_{C} / f_{T}$ ratio, the smallest $J_{C}$ that provides adequate $f_{T}$ should be used.

\section{B. High Breakdown Versus Low Breakdown}

One of the most favorable properties of SiGe HBTs is the low $1 / f$ corner frequency $f_{C}$, which makes them excellent choices for power amplifiers and oscillators [1]. In VCOs operating below the peak cutoff frequency of the HBV device (30 GHz), the HBV device is a better choice than the high $f_{T}$ device. The HBV device has the natural advantage of operating with a larger signal power, thus reducing additive phase noise according to Leeson's theory [11].

A logical question is how the use of the HBV device affects the modulative phase noise up-converted from low-frequency noise. Fig. 5 compares the $S_{I_{B}} \times f$ product as a function of $I_{B}$ for standard and HBV devices on the SiGe control wafer. At the same $I_{B}$, the standard and HBV devices show nearly the same $S_{I_{B}} \times f$ product. Due to their similar $\beta$, the two devices show nearly identical $f_{C}$ at the same $J_{C}$, as shown in Fig. 6. Fig. 7 shows the measured and modeled $f_{C} / f_{T}$ ratio versus $J_{C}$, together with measured $f_{T}$ for both devices. At lower $J_{C}, f_{T}$ is very close in the standard and HBV devices. As $J_{C}$ increases, $f_{T}$ in the HBV devices decreases because of the enhanced Kirk effect due to the low collector doping. This $f_{T}$ difference translates into an $f_{C} / f_{T}$ difference. The $f_{C} / f_{T}$ ratio is very close in the standard and HBV devices before the $f_{T}$ rolloff at high injection. The $f_{C} / f_{T}$ ratio becomes higher in the HBV device after the $f_{T}$ rolloff.

The similar low-injection $1 / f$ noise behavior in the SBV and HBV devices indicates that the $1 / f$ noise sources created by the 


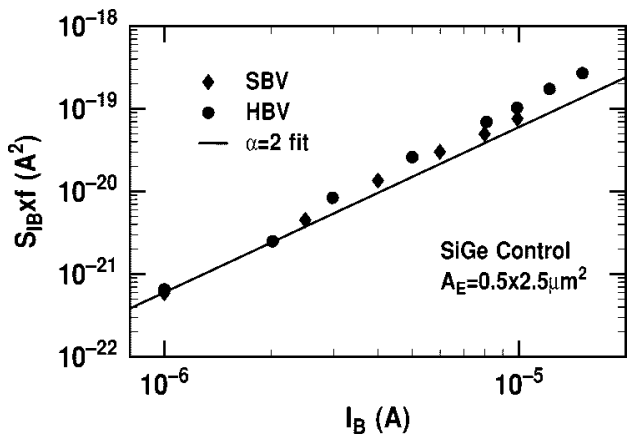

Fig. 5. Measured $S_{I_{B}} \times f$ product as a function of $I_{B}$ for the standard and HBV SiGe control HBTs $\left(A_{E}=0.5 \times 2.5 \mu \mathrm{m}^{2}\right)$.

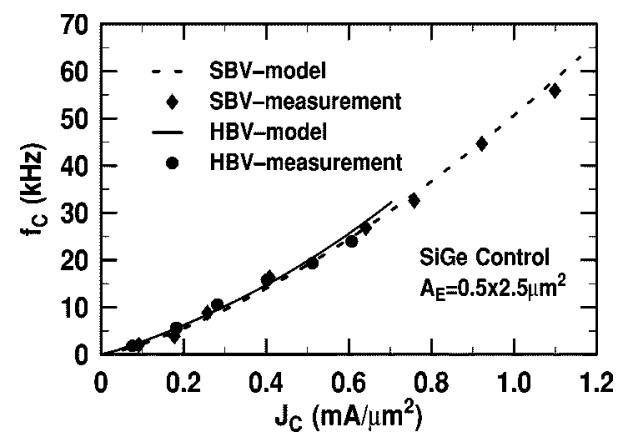

Fig. 6. Measured and modeled $f_{C}$ as a function of $J_{C}$ for the standard and HBV SiGe control HBTs.

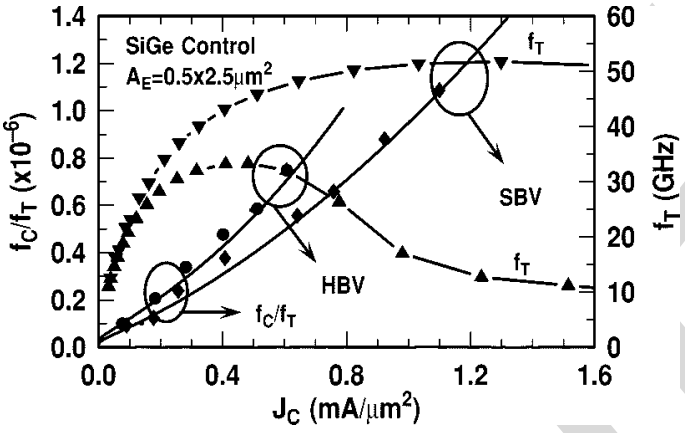

Fig. 7. Measured and modeled $f_{C} / f_{T}$ ratio (left-hand side) and measured $f_{T}$ (right-hand side) for the standard and HBV SiGe control HBTs.

collector implantation through the SiGe base do not contribute significant $1 / f$ noise. Since the up-conversion process is similar in the HBV and SBV devices, we expect the modulative phase noise in the HBV device to be as low as that in the high $f_{T}$ SBV device for current densities lower than the peak $f_{T} J_{C}$.

\section{SiGe Profile Dependence and Profile Design Implications}

The two low-noise SiGe profiles, $L N 1$ and $L N 2$, were optimized to improve $\beta, f_{T}$, and $\mathrm{NF}_{\min }$ without sacrificing $\mathrm{SiGe}$ film stability and peak $f_{T}$ [9], [10]. Fig. 8 shows the measured $f_{T}$ data for the SiGe control, the two low-noise HBTs, and the $\mathrm{Si}$ BJT comparison. All of the SiGe HBTs have much higher $f_{T}$ than the Si BJT. $L N 1$ and $L N 2$ have a slightly higher $f_{T}$ than the SiGe control. The measured $1 / f$ noise $K$ factor is nearly identical for all of the SiGe designs. We thus expect a significant reduction of $f_{C}$, as well as $f_{C} / f_{T}$ in the two low-noise SiGe designs according to (2) and (4).

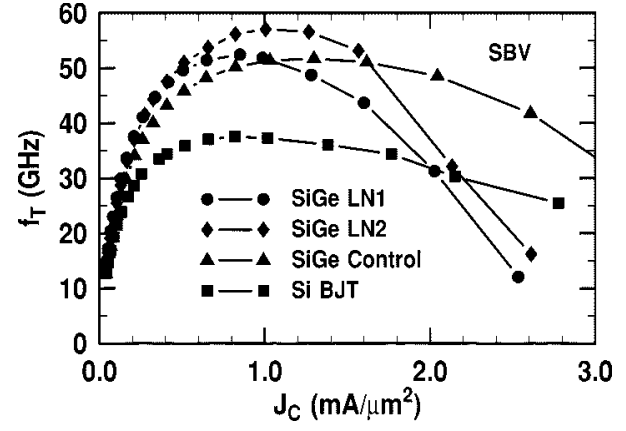

Fig. 8. Measured $f_{T}$ as a function of $J_{C}$ for the SBV Si BJT, SiGe control, and two low-noise HBTs.

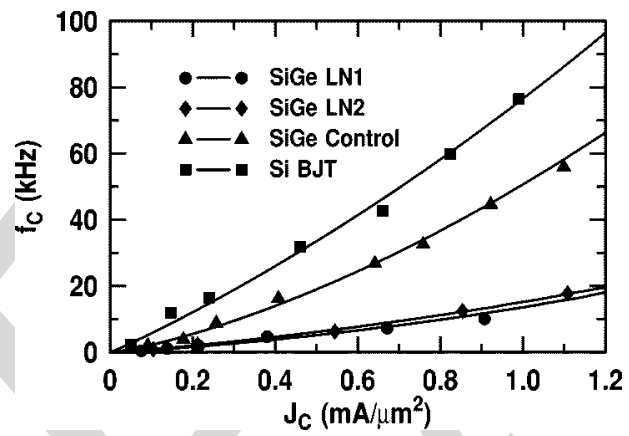

Fig. 9. Measured $f_{C}$ as a function of $J_{C}$ for the SBV Si BJT, SiGe control, and two low-noise HBTs.

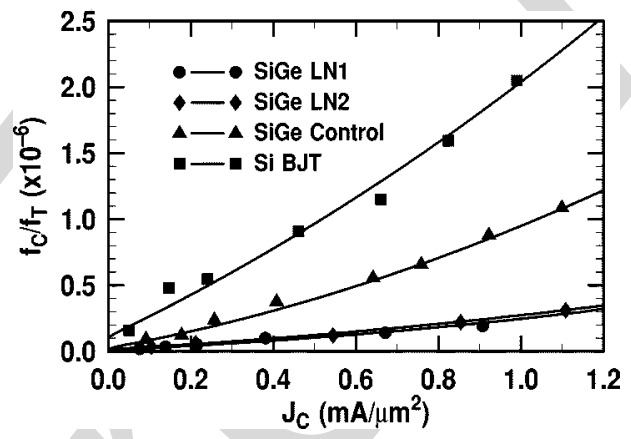

Fig. 10. Measured and modeled $f_{C} / f_{T}$ ratio as a function of $J_{C}$ for the SBV $\mathrm{Si} \mathrm{BJT}, \mathrm{SiGe}$ control, and two low-noise HBTs.

The measured $f_{C}$ data shown in Fig. 9 confirms this expectation. The noise corner frequency $f_{C}$ is indeed the lowest in $L N 1$ and $L N 2$, and highest in the Si BJT. The $f_{C} / f_{T}$ ratio is the lowest in the two low-noise HBT designs because of the much lower $f_{C}$ and slightly higher $f_{T}$, as shown in Fig. 10. For the same operating frequency, SiGe profiles optimized for high $\beta$, and high $f_{T}$ should have better phase noise performance because of the lower $f_{C}$. To achieve the same RF gain, transistors with a higher $f_{T}$ have the advantage to operate at a lower $J_{C}$, which further reduces $f_{C}$ and phase noise.

The above results suggest that the $\tau_{F} / \beta$ ratio can be used as a figure-of-merit for SiGe profile optimization because $f_{C} / f_{T}$ is proportional to $K \tau_{F} / \beta$ according to (4). The $K$ factor is primarily determined by the emitter structure, and independent of the SiGe profile, as well as the collector doping profile, as evidenced by the experimental data. An SiGe profile producing the lowest $\tau_{F} / \beta$ ratio leads to the best $f_{C} / f_{T}$ ratio, and should have the best phase noise performance at higher frequencies. 
The modeled $f_{C}$ and $f_{C} / f_{T}$ ratio for the Si comparison, SiGe $L N 1$, and SiGe $L N 2$ were calculated according to (2) and (4), which were derived using $\alpha=2$. The $\alpha$ for SiGe control (2.24), however, deviates from two. The deviation can be taken into account by using $\alpha$ as a model parameter in the derivation of $f_{C}$ and $f_{C} / f_{T}$. The resulting equations are

$$
\begin{aligned}
f_{C} & =\frac{K I_{B}^{\alpha-1}}{2 q A_{E}} \\
& =\frac{K J_{C}^{\alpha-1}}{2 q A_{E}^{2-\alpha} \beta^{\alpha-1}} \\
\frac{f_{C}}{f_{T}} & =\frac{K}{A_{E}^{2-\alpha}} \frac{\pi}{q} \frac{J_{C}^{\alpha-1}}{\beta^{\alpha-1}}\left(\tau_{F}+V_{t} \frac{C_{t}}{J_{C}}\right) \\
& =\frac{K \pi}{\beta^{\alpha-1} q A_{E}^{2-\alpha}}\left(\tau_{F} J_{C}^{\alpha-1}+V_{t} C_{t} J_{C}^{\alpha-2}\right) .
\end{aligned}
$$

The $K$ factor is now defined using $S_{I_{B}} \times f=K I_{B}^{\alpha} / A_{E}$, and has dimensions of $A^{2-\alpha} \mu \mathrm{m}^{2}$. These modified equations were used to calculate the modeling curve for SiGe control. The modification is necessary to achieve quantitative agreement with measurement for SiGe control. Equations (2) and (4), however, provide better insight and intuitive understanding of the biasing current density dependence because of simple functional forms.

\section{Geometrical Scaling and Optimal Transistor Sizing}

In RFIC design, the device geometry and layout are often used as design variables. The total emitter length required is often physically realized by connecting a number of small area unit cells in parallel. To investigate the low-frequency noise performance of unit cells with different emitter areas, measurements were made on the standard $\mathrm{SiGe}$ control wafer for the four device emitter areas: 1) $A_{E}=0.5 \times 1.0 \mu \mathrm{m}^{2}$; 2) $A_{E}=$ $0.5 \times 2.5 \mu \mathrm{m}^{2}$; 3) $A_{E}=0.5 \times 10 \mu \mathrm{m}^{2}$; and 4) and $A_{E}=$ $0.5 \times 20 \mu \mathrm{m}^{2}$.

Fig. 11 compares the $S_{I_{B}} \times A_{E}$ product as a function of the base current $I_{B}$ for the four devices. SiGe control HBTs of different emitter sizes show the same base current noise and emitter area product $\left(S_{I_{B}} \times A_{E}\right)$ when biased at the same base current. Fig. 12 shows the noise corner frequency $\left(f_{C}\right)$ versus collector current density $\left(J_{C}\right)$ for different emitter areas. To our surprise, the $A_{E}=0.5 \times 2.5 \mu \mathrm{m}^{2}$ device has the lowest $f_{C}$ at the same $J_{C}$. First-order theory predicts the same $f_{C}$ if $\beta$ is assumed to be invariant among different emitter areas. This, however, is not the case in these devices, as shown by the measured $\beta$ versus $J_{C}$ data given in Fig. 13. The $A_{E}=0.5 \times 2.5 \mu \mathrm{m}^{2}$ device has a higher $\beta$ than the other three geometries. The higher $\beta$ for this geometry is consistently observed on both the Si control wafer and the three SiGe HBT wafers, and is possibly due to the strain induced by the shallow trench isolation. $K$ factors and $J_{C}$ being the same, a higher $\beta$ leads to a lower $f_{C}$ because $f_{C}$ is inversely proportional to current gain. On the other hand, for a given $J_{C}, f_{T}$ is independent of geometry before high injection $f_{T}$ rolloff [12]. The $\beta$ difference among different geometries translates into an $f_{C} / f_{T}$ difference. Under the same $J_{C}$, the $A_{E}=0.5 \times 2.5 \mu \mathrm{m}^{2} \mathrm{HBT}$ has the lowest $f_{C}$ and $f_{C} / f_{T}$, therefore, it is an optimum unit cell choice for

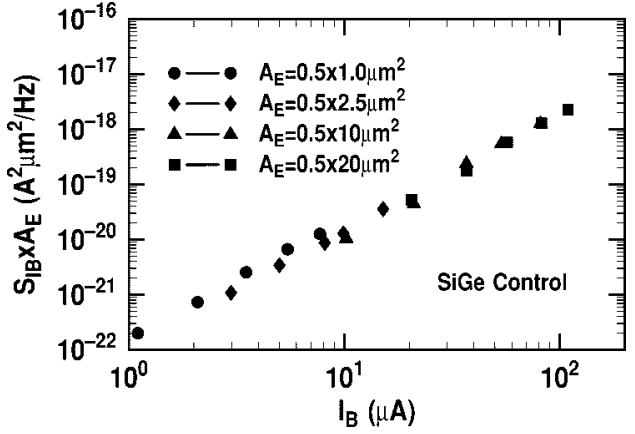

Fig. 11. Measured $S_{I_{B}} \times A_{E}$ versus $J_{C}$ for SiGe control standard breakdown devices with four different emitter areas $\left(A_{E}=0.5 \times 1.0 \mu \mathrm{m}^{2}, 0.5 \times 2.5 \mu \mathrm{m}^{2}\right.$, $0.5 \times 10 \mu \mathrm{m}^{2}$, and $\left.0.5 \times 20 \mu \mathrm{m}^{2}\right)$.

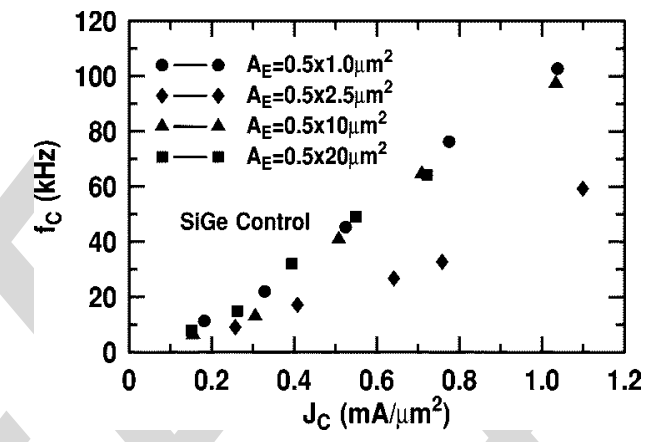

Fig. 12. Measured $f_{C}$ versus $J_{C}$ for $\mathrm{SiGe}$ control standard breakdown devices with four different emitter areas $\left(A_{E}=0.5 \times 1.0 \mu \mathrm{m}^{2}, 0.5 \times 2.5 \mu \mathrm{m}^{2}, 0.5 \times\right.$ $10 \mu \mathrm{m}^{2}$, and $\left.0.5 \times 20 \mu \mathrm{m}^{2}\right)$.

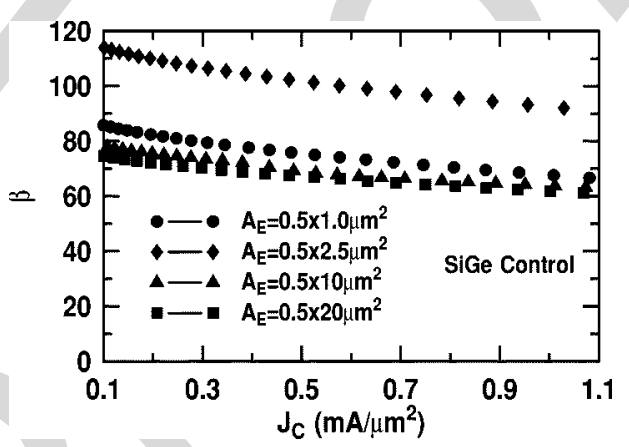

Fig. 13. Measured $\beta$ versus $J_{C}$ for SiGe control standard breakdown devices with four different emitter areas $\left(A_{E}=0.5 \times 1.0 \mu \mathrm{m}^{2}, 0.5 \times 2.5 \mu \mathrm{m}^{2}, 0.5 \times\right.$ $10 \mu \mathrm{m}^{2}$, and $\left.0.5 \times 20 \mu \mathrm{m}^{2}\right)$.

device layout. For instance, if the total effective emitter area required is $A_{E}=0.5 \times 10 \mu \mathrm{m}^{2}$, a parallel combination of four $A_{E}=0.5 \times 2.5 \mu \mathrm{m}^{2}$ HBTs should have a better low-frequency noise performance than ten $A_{E}=0.5 \times 1.0 \mu \mathrm{m}^{2} \mathrm{HBTs}$ in parallel, or one $A_{E}=0.5 \times 10 \mu \mathrm{m}^{2} \mathrm{HBT}$. A corner frequency reduction of nearly $50 \%$ can be achieved by using an optimum unit cell size.

\section{CONCLUSION}

We have presented modeling and experimental results of corner frequency $\left(f_{C}\right)$ and corner frequency to cutoff frequency ratio $\left(f_{C} / f_{T}\right)$ in a commercial SiGe HBT technology. The corner frequency $f_{C}$ is proportional to the collector current density $J_{C}$, and inversely proportional to $\beta$. The $f_{C} / f_{T}$ ratio is 
proportional to the product of $J_{C}$, the forward transit time $\tau_{F}$, the $1 / f$ noise factor $K$, and is inversely proportional to $\beta$. The HBV devices show nearly the same $f_{C}$ and $f_{C} / f_{T}$ ratio as the high $f_{T}$ devices at lower $J_{C}$ prior to the $f_{T}$ rolloff. Measurements of devices featuring various SiGe profile designs show that both $f_{C}$ and the $f_{C} / f_{T}$ ratio can be significantly reduced by careful $\mathrm{SiGe}$ profile optimization without sacrificing $\mathrm{SiGe}$ film stability. The results also suggest that the $\tau_{F} / \beta$ ratio can be used as a $1 / f$ noise figure-of-merit for SiGe profile and collector doping profile optimization in device design. The noise corner frequency was found to be device geometry dependent, and can be reduced by as high as $50 \%$ when the $0.5 \times 2.5 \mu \mathrm{m}^{2} \mathrm{HBT}$ is used as the unit cell in RFIC design.

\section{ACKNOWLEDGMENT}

The authors would like to thank D. Sheridan, <Name of Company/Institution, City, State/Country,> D. Ahlgren, <Name of Company/Institution, City, State/Country,> S. Subbanna, <Name of Company/Institution, City, State/Country,> D. Herman, <Name of Company/Institution, City, State/Country,> B. Meyerson, <Name of Company/Institution, City, State/Country,> and the IBM $\mathrm{SiGe}$ team for their contributions. The wafers were fabricated at IBM Microelectronics, Essex Junction, VT.

\section{REFERENCES}

[1] D. L. Harame, D. C. Ahlgren, D. D. Coolbaugh, J. S. Dunn, G. Freeman, J. D. Gillis, R. A. Groves, F. N. Hendersen, R. A. Johnson, A. J. Joseph, S. Subbanna, A. M. Victor, K. M. Watson, C. S. Webster, and P. J. Zampardi, "Current status and future trends of SiGe BiCMOS technology," IEEE Trans. Electron Devices, vol. 48, pp. 2575-2594, Nov. 2001.

[2] L. S. Vempati, J. D. Cressler, J. A. Babcock, R. C. Jaeger, and D. L. Harame, "Low-frequency noise in UHV/CVD epitaxial Si and SiGe bipolar transistors," IEEE J. Solid-State Circuits, vol. 31, pp. 1458-1467, Oct. 1996.

[3] B. V. Haaren, M. Regis, O. Llopis, L. Escotte, A. Gruhle, C. Mahner, R. Plana, and J. Graffeuil, "Low-frequency noise properties of SiGe HBT's and application to ultra-low phase-noise oscillators," IEEE Trans. Microwave Theory Tech., vol. 46, pp. 647-652, May 1998.

[4] J. Tang, G. Niu, Z. Jin, J. D. Cressler, S. Zhang, A. J. Joseph, and D. L. Harame, "Low-frequency noise figures-of-merit in RF SiGe RF technology," in RFIC Symp. Tech. Dig., Seattle, WA, June 2002, pp. $179-182$.

[5] L. K. J. Vandamme and G. Trefan, "Review of low-frequency noise in bipolar transistors over the last decade," in Proc. IEEE Bipolar/BiCMOS Circuits and Technol. Meeting, 2001, pp. 68-73.

[6] X. Wang, D. Wang, C. Masse, and P. Bacon, "Low phase noise SiGe voltage-controlled oscillators for wireless applications," Microwave J., vol. 45, no. 2, pp. 84-99, Feb. 2002.

[7] S. P. Voinigescu, M. C. Maliepaard, J. L. Showell, G. E. Babcock, D. Marchesan, and M. Schroter, "A scalable high-frequency noise model for bipolar transistors with application to optimal transistor sizing for low-noise amplifier design," IEEE J. Solid-State Circuits, vol. 32, pp. 1430-1439, Sept. 1997.

[8] D. C. Ahlgren, M. Gilbert, D. Greenberg, J. Jeng, J. Malinowski, D. Nguyen-Ngoc, K. Schonenberg, K. Stein, R. Groves, K. Walter, G. Hueckel, D. Colavito, G. Freeman, D. Sunderland, D. L. Harame, and B. Meyerson, "Manufacturability demonstration of an integrated SiGe HBT technology for the analog and wireless marketplace," in Int. Electron Devices Meeting Tech. Dig., 1996, pp. 859-862.

[9] G. Niu, S. Zhang, J. D. Cressler, A. J. Joseph, J. S. Fairbanks, L. E. Larson, C. S. Webster, W. E. Ansley, and D. L. Harame, "SiGe profile design tradeoffs for RF circuit applications," in Int. Electron Devices Meeting Tech. Dig., 1999, pp. 573-576.
[10] _ - "Noise modeling and SiGe profile design tradeoffs for RF applications," IEEE Trans. Electron Devices, vol. 47, pp. 2037-2044, Nov. 2000.

[11] D. B. Leeson, "A simple model of feedback oscillator noise spectrum," Proc. IEEE, vol. 54, pp. 329-330, Feb. 1966.

[12] S. Zhang, G. Niu, J. D. Cressler, A. J. Joseph, G. Freeman, and D. L. Harame, "The effects of geometrical scaling on the frequency response and noise performance of SiGe HBTs," IEEE Trans. Electron Devices, vol. 49, pp. 429-435, Mar. 2002.

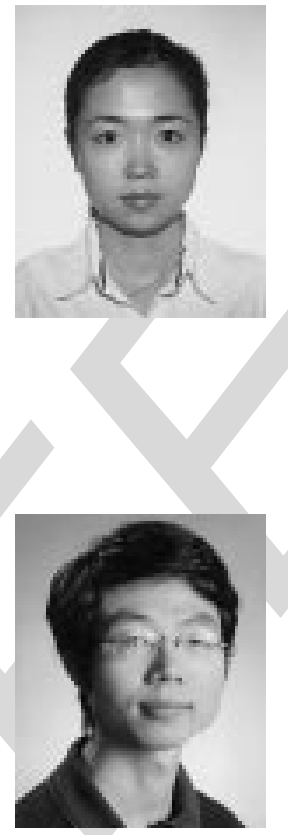

Jin Tang (S'02) was born in Sichuan, China, in November 1977. She received the B.S. degree in electrical engineering from the Huazhong University of Science and Technology, Wuhan, Hubei, China, in 2000, and is currently working toward the M.Sc. degree in electrical and computer engineering at Auburn University, Auburn, AL.

Her research is focused on the modeling and characterization of $1 / f$ noise in SiGe HBT RF technology. Auburn University, Auburn, AL, where he focused on SiGe RF devices. In June 2000, he joined the faculty of Auburn University, where he is currently an Associate Professor of electrical and computer engineering. His research interests include $\mathrm{SiGe}$ devices and circuits, noise, single-event effects, $\mathrm{SiC}$ devices, low-temperature electronics, and <Au. Pls. define TCAD.> TCAD. He has authored or coauthored over 40 journal papers and over 40 conference papers related to his research. He is listed in Who's Who in America.

Dr. Niu served on the Program Committee of the 1997 Asia-South-Pacific Design Automation Conference (ASP-DAC) and currently serves on the Program Committee of the IEEE Bipolar/BiCMOS Circuits and Technology Meeting (BCTM). He has served as a reviewer for many journals, including IEEE ElECtRon DEVICE LETTERS, the IEEE TRANSACTIONS ON ELECTRON DEVICES, the IEEE TRANSACTIONS ON MICROWAVE THEORY AND TECHNIQUES, and IEEE MiCROWAVE AND GUIDED WAVE LETTERS. He was the recipient of the 1993 and 1994 T. D. Lee Physics Award .

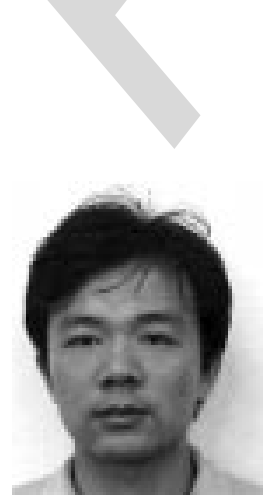

Zhenrong Jin ( $\mathrm{S}^{\prime} 99$ ) was born in Tianjin, China, in 1975. He received the B.S. and M.S. degrees in electrical engineering from Southeast University, Nanjing, China, in 1996 and 1999, respectively, and is currently working toward the Ph.D. degree in electrical engineering at Auburn University, Auburn, AL.

$<$ Au. Affiliation must match first footnote affiliation on page 1 . Are you currently with IBM or at Georgia Tech.?> He is currently an Intern with IBM Microelectronics, Essex Junction, VT, where he is involved with SiGe BiCMOS technology development. He has authored or coauthored several papers in the area of SiGe HBT devices. His current research interests include low-frequency noise in SiGe HBTs, low-noise RF circuits, and SiGe HBT device physics. 


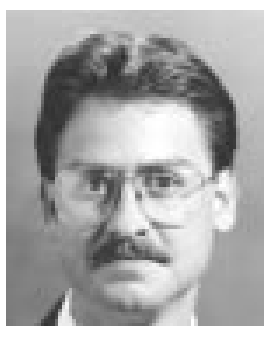

John. D. Cressler (S'86-A'91-SM'91-F'01) received the B.S. degree in physics from the Georgia Institute of Technology, Atlanta, GA, in 1984, and the M.S. and Ph.D. degrees in applied physics from Columbia University, New York, NY, in 1987 and 1990, respectively.

From 1984 to 1992 . he was a member of the research staff at the IBM T. J. Watson Research Center, Yorktown Heights, NY, where he was involved with high-speed $\mathrm{Si}$ and $\mathrm{SiGe}$ bipolar devices and technology. In 1992, he join the faculty at Auburn University, Auburn, AL, where he remained until 2002. When he left Auburn University, he was the Philpott-Westpoint Stevens Distinguished Professor of Electrical and Computer Engineering and Director of the Alabama Microelectronics Science and Technology Center. In 2002, he joined the faculty of the Georgia Institute of Technology, where he is currently a Professor of electrical and computer engineering. His research interests include $\mathrm{SiGe}$ devices and technology, Si-based RF/microwave/millimeter-wave devices and circuits, radiation effects, noise and linearity, cryogenic electronics, $\mathrm{SiC}$ devices, reliability physics, two-dimensional (2-D)/three-dimensional (3-D) device-level simulation, and compact circuit modeling. He has authored or coauthored over 240 technical papers related to his research.

Dr. Cressler was an associate editor for the IEEE JouRnAL OF SOLID-STATE CIRCuITS (1998-2001), and served on the Technical Program Committees of the IEEE International Solid-State Circuits Conference (1992-1998, 1999-2001), the IEEE Bipolar/BiCMOS Circuits and Technology Meeting (1995-1999), the IEEE International Electron Devices Meeting (1996-1997), and the IEEE Nuclear and Space Radiation Effects Conference (1999-2000, 2002). He was the Technical Program chairman of the 1998 International Solid-State Circuits Conference (ISSCC). He currently serves on the Executive Steering Committee for the IEEE Topical Meeting on Silicon Monolithic Integrated Circuits in RF Systems, as international advisor for the IEEE European Workshop on Low-Temperature Electronics, and on the Technical Program Committee for the IEEE International SiGe Technology and Device Meeting. He was appointed an IEEE Electron Device Society Distinguished Lecturer in 1994, and was awarded the 1994 Office of Naval Research Young Investigator Award for his SiGe research program, the 1996 C. Holmes MacDonald National Outstanding Teacher Award by Eta Kappa Nu, the 1996 Auburn University Alumni Engineering Council Research Award, the 1998 Auburn University Birdsong Merit Teaching Award, the 1999 Auburn University Alumni Undergraduate Teaching Excellence Award, and an IEEE Third Millennium Medal in 2000

Shiming Zhang <Au. Pls. give year of student membership.> (S??), photograph and biography not available at time of publication.
Alvin J. Joseph (S'92-M'97) received the B.E. degree in electrical engineering from Bangalore University, Bangalore, India, in 1989, and the M.S. and Ph.D. degrees from Auburn University, Auburn, AL, in 1992 and 1997, respectively, both in electrical engineering. His doctoral research involved the study of physics, optimization, and modeling of cryogenically operated SiGe HBTs.

In 1997, he joined the SiGe Technology Development Group, IBM Microelectronics Division, Essex Junction, VT. He has been involved in various aspects of installing several SiGe BiCMOS technologies into production. He is currently the Process Integration Team Leader for the $0.18-\mu \mathrm{m} \mathrm{SiGe} \mathrm{BiCMOS}$ technology. He has authored and coauthored several technical journal papers and conference publications related to SiGe HBTs.

Dr. Joseph was a member of the Device Physics Subcommittee for the IEEE Bipolar/BiCMOS Circuits and Technology Meeting (BCTM) (1997-2001).

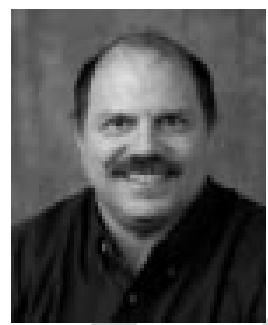

David L. Harame (S'77-M'83-SM'01) was born in Pocatello, ID, in 1948. He received the B.A degree in zoology from the University of California at Berkeley, in 1971, the M.S. degree in zoology from Duke University, Durham, NC, 1973, the M.S degree in electrical engineering from San Jose State University, San Jose, CA, in 1976, and the M.S degree in materials science and the Ph.D. degree in electrical engineering from Stanford University, Stanford, CA, in 1984.

In 1984, he joined the Bipolar Technology Group, IBM T. J. Watson Research Center, Yorktown Heights, NY, where he was involved with the fabrication and modeling of silicon-based integrated circuits. His specific research interests at the IBM T. J. Watson Research Center included silicon and SiGe-channel FET transistors, n-p-n and p-n-p SiGe-based bipolar transistors, complementary bipolar technology, and BiCMOS technology for digital and analog and mixed-signal applications. In 1993, he joined the Semiconductor Research and Development Center (SRDC), Advanced Semiconductor Technology Center (ASTC), IBM, Hopewell Junction, NY, where he was responsible for the development of SiGe BiCMOS technology. $\mathrm{He}$ managed SiGe BiCMOS technology development at the ASTC through 1997. In 1998, he joined IBM's manufacturing organization, Essex Junction, VT, where he managed an $\mathrm{SiGe}$ technology group and installed the $0.5-\mu \mathrm{m} \mathrm{SiGe}$ BiCMOS process in the manufacturing line. In 1999, he rejoined the SRDC, while remaining in Essex Junction, V, T and co-managed the qualification of a $0.25-\mu \mathrm{m}$ SiGe BiCMOS, as well as $0.18-\mu \mathrm{m} \mathrm{SiGe} \mathrm{BiCMOS}$ and two derivative SiGe BiCMOS technologies. In May 2000, he assumed management of the SiGe BiCMOS RF Analog Models and Design Kits area. He currently manages the RF/analog and mixed signal technology, modeling, and design automation areas of the SRDC. He is a Distinguished Engineer of the IBM Corporation.

Dr. Harame is an Executive Committee member of the Bipolar/BiCMOS Circuits and Technology Meeting (BCTM) and a member of the Compact Model Council. 